\title{
ON A THEOREM OF AL'BER ON SPACES OF MAPS
}

\author{
VAGN LUNDSGAARD HANSEN
}

In [1, Theorem 7] (see also [2, Theorem 32]) Al'ber proved

Theorem 1 (Al'ber). Let $V$ be a compact connected Riemannian manifold, and let $M$ be a compact connected Riemannian manifold with strictly negative sectional curvature. Let $C^{2}(V, M)$ denote the space of maps of class $C^{2}$ of $V$ into $M$ equipped with the $C^{2}$-topology. Then one of the following holds for any (path-) component $K$ in $C^{2}(V, M)$ :

(1) $K$ has the homotopy type of a point, and contains a unique harmonic map,

(2) $K$ has the homotopy type of a circle, and all the harmonic maps in $K$ map $V$ with the same value of the Dirichlet integral into the same closed geodesic of $M$,

(3) $K$ has the homotopy type of $M$, and each harmonic map in $K$ maps $V$ into a single point of $M$.

Theorem 1 can also be proved by the methods developped by Eells and Sampson [3] and is very close to being stated explicitely in Hartman [6].

The purpose of this note is to point out that the topological aspect of Theorem 1 is a simple consequence of classical knowledge about the fundamental group of a Riemannian manifold with negative sectional curvature and the following elementary result in homotopy theory.

Lemma. Let $X$ be a locally compact connected $C W$-complex, and $Y$ a space of type $(\pi, 1)$. Let $C(X, Y)$ denote the space of continuous maps of $X$ into $Y$ equipped with the compact-open topology. For any based map $f: X$ $\rightarrow Y$ denote by $C(X, Y ; f)$ the (path-) component in $C(X, Y)$ containing $f$, and denote by $C(\pi ; f)$ the centralizer of $f_{*}\left(\pi_{1}(X)\right)$ in $\pi_{1}(Y)$. Then $C(X, Y ; f)$ is a space of type $(C(\pi ; f), 1)$.

We recall that a connected $C W$-complex $Y$ is called a space of type $(\pi, 1)$, if $\pi$ is a group, $\pi_{i}(Y)=0$ for $i \geq 2$, and $\pi_{1}(Y) \simeq \pi$. We recall also that if $A$ is a subset of the group $G$, then the centralizer of $A$ in $G$ is the subgroup $C_{A}(G)=\{g \in G \mid a g=g a$, all $a \in A\}$.

A proof of the lemma can be found in Gottlieb [4, Lemma 2].

It is a classical result of Hadamard-Cartan that a complete Riemannian manifold $M$ with nonpositive sectional curvature is a space of type $\left(\pi_{1}(M), 1\right)$. From the classical results of Preissmann [8] it also follows that the funda-

Communicated by J. Eells, Jr., October 25, 1975. 
mental group of a compact manifold $M$ with strictly negative sectional curvature has

Property C. A group $\pi$ is said to have property $C$ if any centralizer in $\pi$ is either the identity subgroup, or an infinite cyclic group, or $\pi$.

The necessary Riemannian geometry to prove the results above concerning the fundamental group of a Riemannian manifold with negative sectional curvature can also be found in Gromoll, Klingenberg and Meyer [5, § 7.2].

The following theorem contains the topological aspect of the theorem of Al'ber.

Theorem 2. Let $X$ be a locally compact connected $C W$-complex, and $Y$ a space of type $(\pi, 1)$ where $\pi$ has property $C$. Then any component in $C(X, Y)$ has the homotopy type of either a point, or a circle, or $Y$.

Theorem 2 follows by observing that the component determined by the based map $f: X \rightarrow Y$ is a space of type $(C(\pi ; f), 1)$ and that $C(\pi ; f)$ is either the identity subgroup, or an infinite cyclic group, or $\pi_{1}(Y)$.

We should also remark that the space of maps $C^{2}(V, M)$ has the same homotopy type as $C(V, M)$ by well-known approximation theorems or, for an elegant proof, by Palais [7, Theorem 13. 14].

Al'ber's proof of Theorem 1 involves fairly advanced calculus of variations, and it is necessary for his proof that the domain is compact. So apart from a completely topological setting we obtain also in Theorem 2 a slight generalization of the topological part of Theorem 1 in so far that we only need the domain to be locally compact. It would be interesting to have an example of a manifold $M$, which is a space of type $(\pi, 1)$ where $\pi$ has property $C$, but where $M$ does not admit a Riemannian metric with strictly negative sectional curvature.

\section{References}

[1] S. I. Al'ber, Spaces of mappings into a manifold with negative curvature, Dokl. Akad. Nauk SSSR 178 (1968) 13-16; Soviet Math. Dokl. 9 (1968) 6-9.

[2] - The topology of functional manifolds and the calculus of variations in the large, Russian Math. Surveys 25 (1970) 51-117.

[3] J. Eells \& J. H. Sampson, Harmonic mappings of Riemannian manifolds, Amer. J. Math. 86 (1964) 109-160.

[ 4 ] D. H. Gottlieb, Covering transformations and universal fibrations, Illinois J. Math. 13 (1969) 432-437.

[ 5 ] D. Gromoll, W. Klingenberg \& W. Meyer, Riemannsche Geometrie im Grossen, Lecture Notes in Math. Vol. 55, Springer, Berlin, 1968.

[6] P. Hartman, On homotopic harmonic maps, Canad. J. Math. 19 (1967) 673-687.

[ 7 ] R. S. Palais, Foundations of global non-linear analysis, Math. Lecture Note Series, Benjamin, New York, 1968.

[ 8 ] A. Preissmann, Quelques propriétés globales des espaces de Riemann, Comment. Math. Helv. 15 (1943) 175-216. 